\title{
Electrical breakdown of short multiwalled carbon nanotubes
}

AUTHOR(S):

Tsutsui, Makusu; Taninouchi, Yu-ki; Kurokawa, Shu; Sakai, Akira

\section{CITATION:}

Tsutsui, Makusu ...[et al]. Electrical breakdown of short multiwalled carbon nanotubes. JOURNAL OF APPLIED PHYSICS 2006, 100(9): 094302.

\section{ISSUE DATE:}

2006-11-01

URL:

http://hdl.handle.net/2433/39721

\section{RIGHT:}

Copyright 2006 American Institute of Physics. This article may be downloaded for personal use only. Any other use requires prior permission of the author and the American Institute of Physics. 


\title{
Electrical breakdown of short multiwalled carbon nanotubes
}

\author{
Makusu Tsutsui, ${ }^{\text {a) }}$ Yu-ki Taninouchi, Shu Kurokawa, and Akira Sakai \\ International Innovation Center, Kyoto University, Kyoto 606-8501, Japan
}

(Received 5 January 2006; accepted 18 August 2006; published online 1 November 2006)

\begin{abstract}
Electrical breakdown of short $(\sim 50 \mathrm{~nm})$ multiwalled carbon nanotubes (MWNTs) is studied utilizing mechanically controllable break junction technique with gold electrodes. Measurements of the conductance-bias characteristics near the breakdown point revealed two different types of breakdown behavior for the short MWNTs. In one type designated as type I, the conductance increases nearly linearly with the bias and, over the breakdown point, decreases stepwise. On the other hand, in the type-II breakdown, the conductance shows a transient and irreversible increase right before the breakdown and subsequently jumps to zero. We consider that the type-II breakdown in vacuum is contact related, whereas the type-I breakdown occurs through the usual MWNT heating. (C) 2006 American Institute of Physics. [DOI: 10.1063/1.2364048]
\end{abstract}

\section{INTRODUCTION}

Metallic carbon nanotubes are efficient one-dimensional conductors which can hold an enormous current density as high as $\sim 10^{9} \mathrm{~A} / \mathrm{cm}^{2}$. This high current-carrying capacity of the nanotubes makes them a potential material for interconnects in molecular electronics. ${ }^{1-3}$ Multiwalled carbon nanotubes (MWNTs) are particularly suited to device applications $^{4-6}$ because their large diameter provides small band gaps and facilitates efficient intersubband conduction at high biases. ${ }^{6}$ Furthermore, the concentric shell structure of MWNTs offers a superior mechanical rigidity, ${ }^{7}$ another important characteristic of reliable interconnects. High-field electron transport along MWNTs has thus been a subject of extensive experimental and theoretical studies for the past few years. ${ }^{4,8-15}$

One of the high-field phenomena in MWNTs that is interesting from both academic and practical points of view is a nanotube breakdown induced by high bias/current. The electrical breakdown of MWNTs is indeed a vital issue for their applications to interconnects, since it determines the maximum allowable current through a nanotube. At the same time, various processes involved in the nanotube breakdown, e.g., energy dissipation of hot electrons, local heating and/or oxidation, etc., are of academic interest in their own right. Collins et $a l^{4}{ }^{4}$ investigated the electrical breakdown of substrate-supported MWNTs and revealed that under a constant bias, the current flowing through a nanotube drops discretely with a step of $12-20 \mu \mathrm{A}$. To account for this observation, they proposed a successive shell-by-shell breakdown, where each stepwise current decrement corresponds to the breakdown of one shell (naturally the outermost shell) of the MWNT. After their experiment, the shell-by-shell breakdown has been utilized as a convenient technique for discriminating metallic and semiconducting shells and also for tailoring MWNTs. ${ }^{8}$ Moreover, Collins et al. ${ }^{4}$ showed that the $I-V$ characteristic exhibits a current saturation at high biases and explained it in terms of electron scattering by optical phonon excitations. ${ }^{16-18}$ This suggests that the electron transport

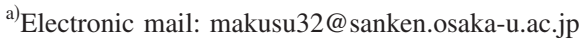

through MWNTs is diffusive at high biases, and the heat dissipation takes place via inelastic electron scattering by optical phonons. In this case, the local temperature along a MWNT would reach the highest in the middle of the nanotube, so that the shell-by-shell breakdown would naturally occur near the midpoint of the nanotube.

On the other hand, Poncharal et al. ${ }^{9}$ carried out high-bias measurements on freestanding MWNTs with their one end being immersed into a $\mathrm{Hg}$ reservoir. They showed that the nanotube failure occurs not at its midpoint but at the MWNT/Hg contact, presumably due to considerable heat dissipation at the contact. ${ }^{10}$ Local heating effects at the nanotube/electrode interface were also inferred in other experiments. ${ }^{19}$ Furthermore, instead of the current saturation, Poncharal and co-workers ${ }^{9,10,14}$ found a linear increase in the conductance with the bias and explained the observed conductance versus bias $(G-V)$ characteristics by assuming a ballistic electron transport in MWNTs. Therefore, there have been two different results on the high-field MWNT breakdown: in one case, the breakdown takes place in the middle of MWNTs, and in another, it occurs at the MWNT/electrode contact. Two latest experiments add further information to the MWNT breakdown. Huang et al. ${ }^{20}$ measured $I-V$ characteristics of freestanding MWNTs specially arranged so that the current flows through all their inner shells. In this way, complexities associated with the shell-to-shell tunneling conduction were eliminated. By carrying out current measurements with simultaneously observing MWNTs with transmission electron microscopy (TEM), they confirmed that the stepwise current decrease definitely corresponds to the shellby-shell breakdown of the nanotube. They also clarified that the shell ablation always takes place in the middle of the nanotube and not at the contact. Thus, their findings show good agreement with those of Collins et al. ${ }^{4}$ Huang et al., ${ }^{20}$ however, observed no current saturation but a linear increase in the conductance with the bias up to $2.5 \mathrm{~V}$. They explained the absence of the current saturation by noting that the large diameter and the short length $(\sim 200 \mathrm{~nm})$ of their MWNTs would change the phonon spectrum and require a higher bias for the onset of substantial electron-phonon scattering than 
that in previous experiment performed on narrower and longer MWNTs. On the other hand, Chiu et al. ${ }^{21}$ carried out breakdown experiments on freestanding MWNTs. For MWNTs longer than $500 \mathrm{~nm}$, they found that the power necessary for the initial shell breakdown scales with $L^{-1}$, with $L$ being the electrode gap distance or equivalently the effective tube length. Also, the electrical breakdown was found to occur at the midpoint of nanotubes, in accordance with Collins et $a{ }^{4}{ }^{4}$ For short MWNTs with $L<500 \mathrm{~nm}$, however, the breakdown power demonstrates little systematic relationship with $L$ and becomes dependent on the nanotube diameter. At the same time, the breakpoint of MWNTs starts to deviate from their midpoint when $L<500 \mathrm{~nm}$. The authors explained the $L$-independent breakdown power by assuming a ballistic phonon transport, which dilutes the heat dissipation and makes the temperature distribution along MWNTs more uniform. The ballistic phonon transport in MWNTs has also been reported in a recent experiment by Brown et al. ${ }^{22}$

These experiments mentioned above clearly indicate that the nanotube length is an important parameter for the electrical breakdown of MWNTs; short and long MWNTs should manifest different breakdown behaviors. Up to this time, most experimental studies of the MWNT breakdown have been made on relatively long nanotubes with $L>100 \mathrm{~nm}$, and little has been understood how short MWNTs with $L$ $<100 \mathrm{~nm}$ undergo electrical breakdown. In the present work, we have exploited the mechanically controllable break junction (MCBJ) technique ${ }^{23,24}$ and experimentally studied the breakdown of 50-nm-long MWNTs. The MCBJ has been widely applied for studying atomic and molecular junctions $^{25,26}$ and thus is best suited for measurements on very short MWNTs as well. In our previous work, we have employed the MCBJ to investigate MWNTs with an effective tube length of $\sim 50 \mathrm{~nm}$ and studied the high-field $G-V$ characteristics and some irreversible effects due to contact heating. ${ }^{27,28}$ To gain deeper insight into the electrical breakdown and the high-field electron transport in MWNTs, we have extended our previous work to cover even higher biases and examined the breakdown characteristics.

\section{EXPERIMENT}

As in our previous studies, ${ }^{27,28}$ we employed the MCBJ in our nanotube breakdown experiments. Operating principles and other details of the MCBJ technique can be found in Refs. 23 and 24. We used a 0.5-mm-diameter Au wire and broke it to form a pair of nanogapped electrodes. Bridging of a single MWNT over the electrodes was accomplished in the following manner. We start with preparing a MWNTdispersed solution by sonicating chemical-vapor-deposition (CVD)-grown MWNT raw materials (Sigma-Aldrich, Inc.) in ethanol. Scanning electron microscopy (SEM) observations on MWNTs after sonication showed that they consist of tubes with diameters ranging from 5 to $20 \mathrm{~nm}$ with an average length of $\sim 2 \mu \mathrm{m}$. We then put a drop of the solution onto the $\mathrm{Au}$ electrodes and repeatedly open and close the junction until a long conductance plateau can be observed during the electrode opening. When such a plateau appears at $(0.1-1) G_{0}\left(G_{0} \equiv 2 e^{2} / h\right.$ is the quantum unit of conductance) and continues for the gap opening of $>100 \mathrm{~nm}$, it unambiguously signifies the bridging of a MWNT. Note that the nanotube is only supported by Au electrodes, and hence it is in a freestanding configuration. The samples prepared in this manner usually exhibit the same conductance characteristics as those reported on single MWNTs. Therefore, the bridging of MWNT bundles seems unlikely, though its possibility cannot be completely ruled out. Besides, we could detect a breakdown characteristic which can be attributed to a bundle of MWNTs, as will be mentioned in Sec. III A, and discriminate such breakdowns from those of single MWNTs.

After bridging a MWNT, the electrode gap separation was adjusted to $L<50 \mathrm{~nm}$. Then, we increased the dc bias from $0.1 \mathrm{~V}$ at a rate of $\sim 1 \mathrm{~V} / \mathrm{s}$ and measured the $G-V$ characteristic until the nanotube breaks down. Ethanol evaporates well before starting the breakdown measurements. All measurements were made at room temperature in air or in medium vacuum of $\sim 2 \times 10^{-5}$ Torr.

\section{RESULTS AND DISCUSSION}

\section{A. High-bias $G-V$ characteristics}

Figure 1 shows three typical $G-V$ curves of MWNTs observed in air [(a) and (b)] and in vacuum [(c)]. The corresponding $I-V$ curves are also displayed in insets for reference. In this paper, we prefer to discuss $G-V$ rather than $I-V$ curves because the irreversible behaviors under high biases, which will be mentioned shortly, can be observed more clearly in the $G-V$ curves. We also note that some samples exhibit complicated features, e.g., consecution of two different $I-V$ curves separated by a large current drop. We judged that such characteristics are due to the bridging of a MWNT bundle and did not take them into consideration.

At low biases below $1.5 \mathrm{~V}$, the conductance increases almost linearly with the bias in agreement with our previous observations. ${ }^{27}$ When the bias exceeds $1.5-2.0 \mathrm{~V}$, the $G-V$ curve starts to deviate from its linear behavior and becomes nonlinear. In this bias regime, the conductance often demonstrates a few small stepwise increments as seen in $G-V$ curves in Fig. 1. These conductance jumps will be discussed later. On the verge of the electrical breakdown, the measured $G-V$ characteristics exhibit two distinct behaviors. In one case, the conductance shows little transient increase and, after the breakdown, drops to zero in a stepwise manner. An example of this type of $G-V$ curve is illustrated in Fig. 1(a). The discrete decrement of the conductance unambiguously signifies the shell-by-shell breakdown of the MWNT. ${ }^{4,8,20}$ To distinguish this type of breakdown from another one, we designate it as "type-I" breakdown. In another case, referred to as "type-II" breakdown, the conductance exhibits a steep and nonlinear upshoot right before the breakdown and subsequently drops to zero almost instantaneously without showing any fine structures. ${ }^{29}$ Two $G-V$ curves shown in Figs. 1(b) and 1(c) represent this type-II breakdown observed in vacuum and in air, respectively. ${ }^{30}$ Note that these two $G-V$ curves are similar in shape but have different conductance scales. Generally, MWNTs measured in air tend to fail at lower conductance than those tested in vacuum. 

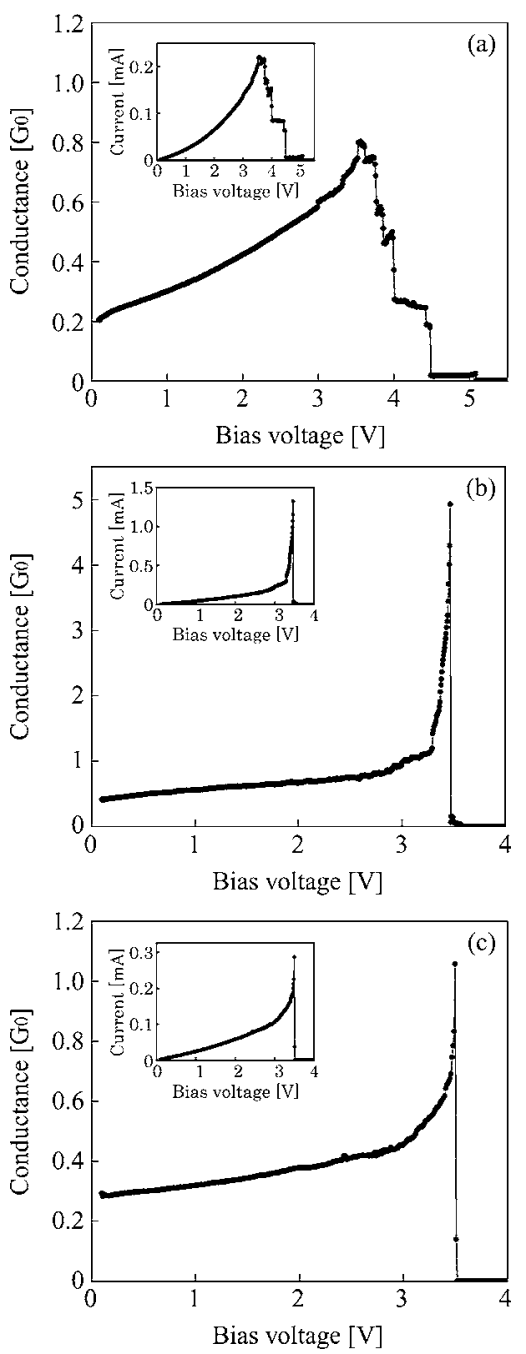

FIG. 1. Two types of MWNT breakdown observed in this experiment. In the type-I breakdown, the $G-V$ curve shows little nonlinearity up to the breakdown point and exhibits stepwise drops of the conductance during the electrical breakdown (a). On the other hand, the type-II breakdown is characterized by the sharp transient increase in the $G$ - $V$ curve, which appears in both $G-V$ curves obtained in a vacuum (b) and in air (c). The insets are the $I-V$ characteristics corresponding to each $G-V$ curve.

A similar sharp increase in the conductance near the MWNT breakdown was also reported in previous literatures, though its origin remains unclarified. ${ }^{21,31}$ To obtain some insights on the transient increase in the conductance, we first measured the $G-V$ curve in the prebreakdown regime and then repeated $G-V$ measurements with each time slightly increasing the upper limit of the bias range and approaching the breakdown point. The same procedure was employed in our previous work on high-bias heating effects on MWNT/Au contacts, and some technical details can be found therein. ${ }^{28}$ Figure 2 displays a typical result of such repeated $G-V$ measurements conducted on a MWNT in vacuum. As seen in the inset, the $G-V$ curve is almost completely reversible for biases $<2.5 \mathrm{~V}$. When the bias range is further extended, however, a sudden increase in the conductance emerges, the occurrence of which seems to be highly bias dependent. After this small conductance jump, the $G-V$ curve is nearly the same as before but slightly shifted upward (Fig. 2, inset). When we further approach the breakdown

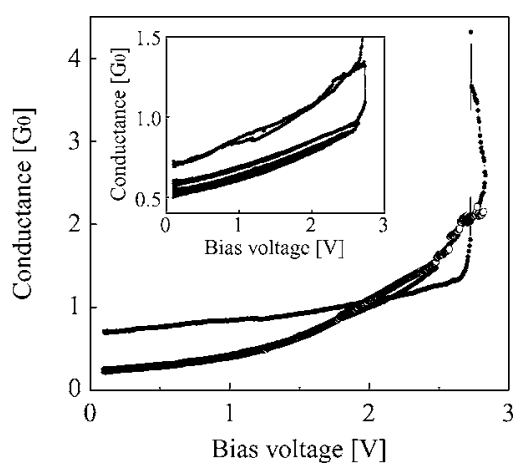

FIG. 2. A result of the stepwise $G-V$ measurement conducted in a vacuum. Small conductance steps seen in the inset are indicative of modifications of MWNT/Au contacts. After the transient increase of the conductance, the $G-V$ curve became nonlinear, perhaps due to partial ablation of the outermost shell of the nanotube.

point, there suddenly occurs a rapid increase in the conductance. Once this happens, the sharp rise in the conductance continues even when we decrease the bias. After this transient upshoot, the conductance completely changes its bias dependence. Instead of its linear behavior, the $G-V$ curve becomes strongly nonlinear even in the low bias regime below $2.5 \mathrm{~V}$. As a result, the new $G-V$ curve crosses the initial one, and the conductance becomes lower near zero bias but higher at $>2.5 \mathrm{~V}$ compared to conductance values before the transient upshoot. This nonlinear $G-V$ characteristic was found reproducible for the forward bias sweep, and the linear $G-V$ curves no longer appeared.

The observed rapid increase in conductance and the associated transition from the linear to the nonlinear $G-V$ characteristics clearly indicate that the specimen undergoes certain irreversible changes right before the breakdown. Since we have no probing tools with our MCBJ, we cannot know exactly what kind of changes they are and where they take place. Nevertheless, some implications can be obtained by comparing our results with previous observations. A similar rapid increase in conductance has been reported by Chiu $e t$ al. ${ }^{21}$ which was attributed to the structural annealing in MWNT. It is, however, not clear whether the $G-V$ characteristic makes irreversible changes after the rapid conductance increment. On the other hand, the linear to nonlinear change in $G-V$ characteristics has been observed on MWNTs which suffered substantial structural damages via partial shell ablations. ${ }^{31}$ According to Poncharal and co-workers, ${ }^{9,10}$ such a partial ablation usually occurs at the electrode-MWNT contacts. We further note that Javey et al. ${ }^{18}$ observed a permanent change in electric properties of short $(<100 \mathrm{~nm})$ singlewalled carbon nanotubes after applying high biases $(>1 \mathrm{~V})$, which could be due to contact degradation. It is thus likely that the observed transient increase in conductance and the irreversible change in the $G-V$ curve are due to a contact modification under high biases/currents and a subsequent partial ablation of the outermost shell in touch with one of two Au electrodes. If this is the case, the current path after the ablation would be Au-(inner shells)-(outermost shell)-Au and would involve the intershell conduction. At low biases, the intershell barrier for electrons tends to reduce the conductance compared to its value before the ablation, while 
under high biases, the barrier becomes less impeditive, and the conductance is enhanced due to the improved contact of the inner shell to the Au electrode. Thus, the partial breakdown of MWNT is consistent with the nonlinear $G-V$ curve in Fig. 2 which, at low biases, falls below the one before the conductance transient but exceeds it at higher biases. Chung et $a l^{31}$ discussed that the oxygen adsorption on inner shells should make some contribution for enhancing the conductance of partially ablated MWNTs. Though we found little substantial difference between the conductance transients of MWNTs in air and in vacuum, the quality of our vacuum is not sufficient to rule out the possibility of oxygen adsorption, and further work is required to observe the effects of gas adsorption on the electrical breakdown.

The type-II breakdown is characterized by the conductance transient discussed above, which we consider due to the contact modification. In our experiment, the nanotube immediately failed after the conductance transient during the type-II breakdown. ${ }^{29}$ Another breakdown, similar to the one in the type I, might also be possible after the conductance transient when the contact modification acts to uniform the heating distribution. It is thus not unexpected that Chiu et $a .^{21}$ reported a breakdown which shows the conductance transient and the subsequent stepwise breakdown. The behavior after the conductance transient is likely to depend on the details of the metal/MWNT contacts. On the other hand, the irreversible conductance transient has not been observed for longer MWNTs and can be considered as characteristic of short MWNTs comparable to the electron (optical)-phonon scattering length. Even for short MWNTs, our experimental results showed that some tubes undergo the type-I breakdown without the conductance transient. The cause of this distinction between the type I and the type II is not clear at this time. One possibility would be the tube diameter. As suggested by Svizhenko and Anantram, ${ }^{32}$ smaller-diameter tubes tend to have more uniform potential distribution and hence more homogeneous heat dissipation, which leads to the type-I breakdown. Measurements on diameter-selected MWNTs would clarify the issue.

\section{B. Breakdown voltage and power for MWNTs}

We have noticed that MWNTs exhibiting the type-II breakdown tend to fail at nearly the same bias voltage, as seen in Figs. 1(b) and 1(c), though the magnitude of their peak conductance $G_{p}$ is much different. Figure 3 shows values of the breakdown voltage $V_{p}$ obtained on MWNTs in vacuum and in air, respectively. The constancy of $V_{p}$ is particularly evident for the type-II breakdown in vacuum, even though the magnitude of $G_{p}$ is widely distributed from $\sim 0.3 G_{0}$ to $\sim 20 G_{0}$ (corresponding to peak currents $I_{p}$ ranging from $70 \mu \mathrm{A}$ to $4.6 \mathrm{~mA})$. In the case of MWNTs in air, the $V_{p}$ values show some scatter, but still have an average close to the constant $V_{p}$ in vacuum. We note that a similar constant breakdown voltage has been reported on the current-induced break of metal nanocontacts. ${ }^{33,34}$ Electromigration of metal atoms are often inferred as a possible break mechanism, but details are still under investigation. ${ }^{35}$ For MWNTs, we previously observed that the high-bias conduc-

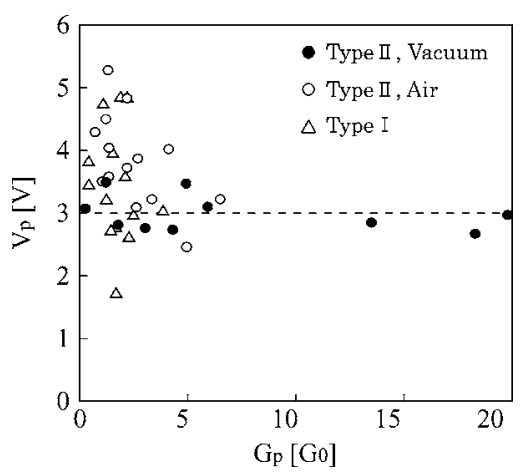

FIG. 3. The peak bias voltage $V_{p}$ is plotted against the maximum conductance $G_{p}$ for MWNTs that have undergone the type-II breakdown. The data points nicely lie on a horizontal straight line for the type II in vacuum, indicating that the conductance attains a peak at a constant bias $\sim 3 \mathrm{~V}$. On the other hand, $V_{p}$ obtained in air shows some scatter.

tance shows two-level fluctuations (TLFs) that can be attributed to moving $\mathrm{Au}$ atoms of electrodes. Thus, electrode atoms at the contact would be mobile in the high-bias regime, and their massive migration is likely involved in the contact modification during the irreversible conductance increment observed in the type-II breakdown. It is, however, unclear, as in the break of metal nanocontacts, whether the electromigration is a primary breakdown mechanism of MWNTs and how it gives rise to the constant break voltage. More data, on the starting temperature of electromigration ${ }^{35}$ and the potential buildup at the contacts, ${ }^{36}$ for example, would be required for properly assessing the role of electromigration in the MWNT breakdown.

The breakdown power, the power dissipated at the breakdown point, is defined as $P_{b} \equiv G_{p} V_{p}^{2}$. For the type-I and the type-II breakdowns, we plot $P_{b}$ as a function of the lowbias resistance $R_{0}$ of each MWNT in Fig. 4. Here, $R_{0}$ is an alternative of the contact resistance which cannot be measured with the two-terminal MCBJ. Logarithmic scales are used for $P_{b}$ and $R_{0}$, respectively. As seen in the figure, $P_{b}$ of the type-I breakdown reveals no obvious correlation with $R_{0}$. On the other hand, $\log P_{b}$ for the type-II breakdown, observed in vacuum, shows a linear correlation with $\log R_{0}$, suggesting a power-law dependence $P_{b} \propto R_{0}^{\alpha}$ with $\alpha \sim-1.1$. The presence of the $P_{b}-R_{0}$ correlation is consistent with our

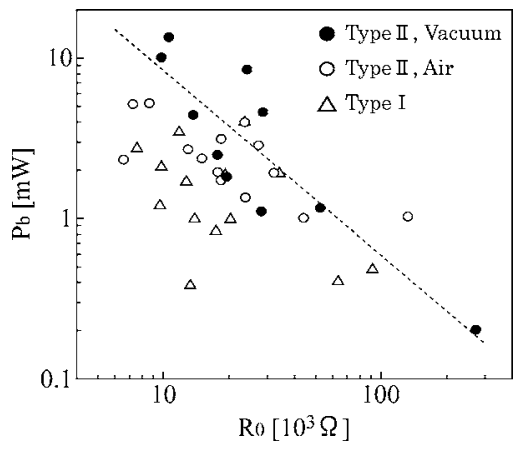

FIG. 4. Log-log correlation between the breakdown power $P_{b} \equiv G_{p} V_{p}^{2}$ and the low-bias resistance $R_{0}$ for MWNTs showing the type-I and the type-II breakdowns. The dashed line is a power-law fit $P_{b} \propto R_{0}^{\alpha}$ to the plot obtained in vacuum with $\alpha \sim-1.1$, which reveals a certain correlation between the $P_{b}$ for the type-II breakdown and the $R_{0}$. 
interpretation that the transient conductance increase, observed in the type-II breakdown, is due to the contact modification. If the breakdown occurs at the contact, the power would be dissipated at the contact and approximated as $P_{b}$ $\sim V_{p}^{2} / R_{c} \sim V_{p}^{2} / R_{0}$. For the type-II breakdown in vacuum, $V_{p}$ $\sim 3 \mathrm{~V}$, as shown in Fig. 3. Then, we have $P_{b} \propto R_{0}^{-1}$, in qualitative accordance with the observed relation $P_{b} \propto R_{0}^{-1.1}$. Thus, the experimental $P_{b}-R_{0}$ relation also implies the contactrelated breakdown for the type-II breakdown in vacuum.

Situations are less clear for the type-II breakdown in air; $V_{p}$ shows more scatter than that in vacuum in Fig. 3, and the $P_{b}-R_{0}$ correlation appears less identifiable in Fig. 4. For MWNTs in air, their breakdown may be affected by the tube oxidation $^{4}$ or the solvent residues left adsorbed at or in the vicinity of the MWNT/Au contacts. However, the $P_{b}$ in air for $R_{0}>\sim 40 \mathrm{k} \Omega$ exceeds that in vacuum so that these factors do not necessarily promote the breakdown. Also, as we noted before, the characteristic conductance increase shows little difference between that in air and in vacuum. At this time, we have no clear-cut explanation for the $V_{p}$ and $P_{b}$ in air.

For the type-I breakdown, the absence of the $P_{b}-R_{0}$ correlation suggests that the electrical breakdown is not related to contact properties but probably caused by homogeneous heat dissipation along MWNTs due to substantial electronphonon scattering at high biases. ${ }^{4,8,20}$ For freestanding MWNTs with lengths shorter than $500 \mathrm{~nm}$, Chiu et al. ${ }^{21} \mathrm{re}-$ ported that the breakdown power varies with the tube diameter $D$ as $P_{b} \propto D^{2.1}$. When we simply apply this relation to our $P_{b}=(0.4-4) \mathrm{mW}$, we obtain tube diameters in the range of 5-15 $\mathrm{nm}$. Since these diameters are not much different from those estimated from our SEM observations, the scatter in the $P_{b}$ data is presumably due to variations in the tube diameter. All these results suggest that the type-I breakdown is primarily due to heat dissipation caused by substantial electron-phonon scattering inside the MWNTs.

\section{SUMMARY}

We have investigated the electrical breakdown of MWNTs bridging over nanogaps of $<50 \mathrm{~nm}$ in separation provided with the MCBJ technique. We found two distinct high-bias breakdown behaviors, which we designate as the type-I and the type-II breakdowns. The type-I breakdown typically reveals the stepwise conductance drops suggesting the shell-by-shell breakdown. The breakdown power $P_{b}$ shows little correlation with the low-bias resistance $R_{0}$. We consider that the type-I breakdown occurs at the MWNT itself and is caused by homogenous heat generation due to substantial electron-phonon scattering inside the nanotube.

The type-II breakdown is characterized by the steep prebreakdown increase in conductance, which accompanies irreversible changes in the $G-V$ characteristics. The breakdown power in vacuum varies as $P_{b} \propto 1 / R_{0}$. The irreversible conductance changes and the $P_{b}-R_{0}$ correlation suggest that the type-II breakdown is contact-related and occurs at MWNT/Au contacts. Presumably, the short effective length of our MWNTs, comparable to the electron-phonon scatter- ing length, would lead to the local heat dissipation near the contact and would make it possible to observe the contactrelated breakdown.

${ }^{1}$ B. Q. Wei, R. Vajtai, and P. M. Ajayan, Appl. Phys. Lett. 79, 1172 (2001).

${ }^{2}$ F. Kreupl, A. P. Graham, G. S. Duesberg, W. Steinhogl, M. Liebau, E. Unger, and W. Honlein, Microelectron. Eng. 64, 399 (2002).

${ }^{3}$ P. L. McEuen, M. S. Fuhrer, and H. Park, IEEE Trans. Nanotechnol. 1, 78 (2002).

${ }^{4}$ P. G. Collins, M. Hersam, M. Arnold, R. Martel, and Ph. Avouris, Phys. Rev. Lett. 86, 3128 (2001).

${ }^{5}$ A. P. Graham, G. S. Duesberg, R. V. Seidel, M. Liebau, E. Unger, W. Pamler, F. Kreupl, and W. Hoenlein, Small 1, 382 (2005).

${ }^{6}$ H. J. Li, W. G. Lu, J. J. Li, X. D. Bai, and C. Z. Gu, Phys. Rev. Lett. 95, 086601 (2005).

${ }^{7}$ C. Schönenberger, A. Bachtold, C. Strunk, J.-P. Salvetat, and L. Forró, Appl. Phys. A: Mater. Sci. Process. 69, 283 (1999).

${ }^{8}$ P. G. Collins, M. S. Arnold, and Ph. Avouris, Science 292, 706 (2001).

${ }^{9}$ P. Poncharal, C. Berger, Y. Yi, Z. L. Wang, and W. A. de Heer, J. Phys. Chem. B 106, 12104 (2002).

${ }^{10}$ C. Berger, Y. Yi, P. Poncharal, and W. A. de Heer, New J. Phys. 5, 158 (2003).

${ }^{11}$ Y. X. Liang, Q. H. Li, and T. H. Wang, Appl. Phys. Lett. 84, 3379 (2004).

${ }^{12}$ B. Bourlon, D. C. Glattli, B. Placais, J. M. Berroir, C. Miko, L. Forro, and A. Bachtold, Phys. Rev. Lett. 92, 026804 (2004).

${ }^{13}$ Y. Hamada, H. Negishi, S. Akita, and Y. Nakayama, Jpn. J. Appl. Phys., Part 1 44, 1629 (2005).

${ }^{14}$ S. Frank, P. Poncharal, Z. L. Wang, and W. A. de Heer, Science 280, 1744 (1998).

${ }^{15}$ M. P. Anantram, Phys. Rev. B 62, R4837 (2000).

${ }^{16}$ Z. Yao, C. L. Kane, and C. Dekker, Phys. Rev. Lett. 84, 2941 (2000).

${ }^{17}$ J.-Y. Park et al., Nano Lett. 4, 517 (2004).

${ }^{18}$ A. Javey, J. Guo, M. Paulsson, Q. Wang, D. Mann, M. Lundstrom, and H. Dai, Phys. Rev. Lett. 92, 106804 (2004).

${ }^{19}$ M. M. Yazdanpanah, S. Chakraborty, S. A. Harfenist, R. W. Cohn, and B. W. Alphenaar, Appl. Phys. Lett. 85, 3564 (2004).

${ }^{20}$ J. Y. Huang, S. Chen, S. H. Jo, Z. Wang, D. X. Han, G. Chen, M. S. Dresselhaus, and Z. F. Ren, Phys. Rev. Lett. 94, 236802 (2005).

${ }^{21}$ H.-Y. Chiu, V. V. Deshpande, H. W. Ch. Postma, C. N. Lau, C. Miko, L. Forro, and M. Bockrath, Phys. Rev. Lett. 95, 226101 (2005).

${ }^{22}$ E. Brown, L. Hao, J. C. Gallop, and J. C. Macfarlane, Appl. Phys. Lett. 87, 023107 (2005).

${ }^{23}$ C. J. Muller, J. M. van Ruitenbeek, and L. de Jongh, Physica C 191, 485 (1992).

${ }^{24}$ C. J. Muller, J. M. van Ruitenbeek, and L. de Jongh, Phys. Rev. Lett. 69, 140 (1992).

${ }^{25}$ M. Reed, C. Zhou, C. Muller, T. Burgin, and J. Tour, Science 278, 252 (1997).

${ }^{26}$ R. H. M. Smit, Y. Noat, C. Untiedt, N. D. Lang, M. C. van Hermert, and J. M. van Ruitenbeek, Nature (London) 419, 906 (2002).

${ }^{27}$ M. Tsutsui, Y. Taninouchi, S. Kurokawa, and A. Sakai, Nanotechnology 16, 1863 (2005)

${ }^{28}$ M. Tsutsui, Y. Taninouchi, S. Kurokawa, and A. Sakai, Jpn. J. Appl. Phys., Part 1 45, 341 (2006)

${ }^{29}$ The type-II breakdown takes place "instantaneously" within temporal resolution of our conductance measurements, which is typically $50 \mathrm{~ms}$. We carried out a couple of fast conductance measurements, sacrificing the conductance sensitivity, where the type-II breakdown showed no stepwise structures within a time resolution of $0.5 \mu \mathrm{s}$.

${ }^{30}$ Out of 28 MWNTs measured in air, 14 tubes demonstrated this rapid increase in the conductance right before the breakdown. In vacuum, the fraction is 10 out of 14 MWNTs.

${ }^{31}$ J. Chung, K.-H. Lee, J. Lee, D. Troya, and G. C. Schatz, Nanotechnology 15, 1596 (2004)

${ }^{32}$ A. Svizhenko and M. P. Anantram, Phys. Rev. B 72, 085430 (2005).

${ }^{33}$ W. Y. Jang, N. N. Kulkarni, C. K. Shih, and Z. Yao, Appl. Phys. Lett. 84, 1177 (2004).

${ }^{34}$ H. Park, A. K. L. Lim, A. P. Alivosatos, J. Park, and P. L. McEuen, Appl. Phys. Lett. 75, 301 (1999).

${ }^{35}$ A. Fujii, M. Tsutsui, S. Kurokawa, and A. Sakai, Phys. Rev. B 72, 045407 (2005).

${ }^{36}$ M. L. Trouwbrost, S. J. van der Molen, and B. J. van Wees, e-print condmat/0510385. 